\title{
COMMENT
}

\section{Are we there yet? Advanced technologies for young children with type 1 diabetes: comment in response to "Type 1 diabetes mellitus management in young children: implementation of current technologies"}

\author{
Mark A. Clements (iD), Susana R. Patton ${ }^{2}$, Ryan J. McDonough ${ }^{1}$ and Michael Artman ${ }^{1}$ \\ Pediatric Research (2020) 87:616-618; https://doi.org/10.1038/s41390-020-0787-8
}

The paper by Nevo-Shenker et al. ${ }^{1}$ presents the evidence available to support use of recently developed diabetes self-management technologies in very young children (<age 6-years-old) with type 1 diabetes (T1D). The specific technologies the authors write about include continuous glucose monitors (CGMs), continuous subcutaneous insulin infusion (CSII; aka insulin pump) therapy, sensoraugmented pump (SAP) therapy, predictive low-glucose suspend (PLGS) technology, and hybrid closed loop $(\mathrm{HCL})$ insulin delivery. While the authors present promising early evidence for each technology, the limited number of studies, the small samples of young children in those studies, the limited cognitive and behavioral phenotyping of the children and caregivers in the studies, the lack of technologies designed specifically for young children, and the continued lack of well-designed longitudinal randomized clinical trials specifically addressing safety and efficacy in young children remain problematic. Collectively, these limitations constitute critical gaps in our approaches to optimizing care for young children with T1D and evoke multiple research questions that must be explored.

\section{LIMITATIONS TO EXISTING RESEARCH}

\section{Outcomes that matter}

Recent studies highlight the limitations of hemoglobin A1c $(\mathrm{HbA1c})$ as a biomarker of disease control. HbA1c relates strongly to recent average daily glucose levels, and through multiple historic trials, the evidence validates its importance as a proxy for risk of future health complications. Yet, as measures of disease control, there may be less bias in an individual's average daily glucose levels, time in range, time spent hyper- and hypoglycemic, and glycemic variability derived from CGM data, and these measures may equally relate to future outcomes. It is also important that researchers, regulatory agencies, and policymakers consider the importance of non-glycemic outcomes. Young children can experience diabetic ketoacidosis (DKA), which is associated with significant in-hospital morbidity. The prevalence of severe hypoglycemia $(\mathrm{SH})$ is also highest among very young children compared to older youth, ${ }^{2}$ and evidence suggests that $\mathrm{SH}$ can detrimentally impact cognitive development in young children. ${ }^{3}$ Similarly, hypoglycemia fear (FH) occurs frequently among parents of young children and evidence suggests that $\mathrm{FH}$ can impact parent mental health, affect sleep quality, adherence to the treatment plan, family quality of life, and family functioning. ${ }^{4,5}$ Finally, many individuals with diabetes and clinicians have endorsed the importance of optimizing quality of life (QOL) among children and families. ${ }^{6}$

Continuous subcutaneous insulin infusion

As the authors point out, CSII associates with improved disease control in very young children in some but not all studies; whether it associates with reduced SH or DKA requires further study. ${ }^{7}$ Nevo-Shenker et al. ${ }^{1}$ also report an association between CSIl therapy and reductions in FH in several prior studies. One study (reference \#36) revealed reductions in FH-related worry with CSII, but not in FH-related behavior among caregivers. Unfortunately, several of these studies reporting $\mathrm{FH}$ among caregivers of young children included the variable as a secondary outcome and failed to use a valid measure of FH. However, one study that did use a valid measure of $\mathrm{FH}$, but was not referenced by the authors, reported a moderate amount of $\mathrm{FH}$ in parents of children using CSII. ${ }^{8}$ Nevo-Shenker et al. ${ }^{1}$ appropriately point out that further studies are needed to determine how caregiver $\mathrm{FH}$ relates to glycemic control in young children with T1D, and that only small retrospective studies have reported on barriers to insulin pump use and factors contributing to insulin pump discontinuation in very young children.

Continuous glucose monitor

CGM detects hypoglycemia better than self-monitoring blood glucose (SMBG) among young children with T1D. Studies suggest associations between CGM therapy and improved A1C in this population. ${ }^{9}$ Qualitative interview data from the recent SENCE trial indicate that parents feel less worried about glucose excursions when their children use $\mathrm{CGM}^{10}{ }^{10}$ but quantitative studies report conflicting results on whether CGM reduces parent FH. Clinicians nearly ubiquitously recognize additional barriers and problems related to CGM therapy in young children. These include issues with skin sensitivity and skin breakdown, as well as issues related to parents' constant access to the highly granular moment-bymoment data that some CGM devices can share remotely. Existing research has not defined predictors of success or failure on this therapy; knowledge of predictors would enable investigators to

${ }^{1}$ Children's Mercy Hospital, Kansas City, MO, USA and ${ }^{2}$ Nemours Children's Health System, Jacksonville, FL, USA

Correspondence: Mark A. Clements (maclements@cmh.edu)

Received: 27 December 2019 Accepted: 8 January 2020

Published online: 29 January 2020 
design behavioral and educational interventions to optimize the odds of success among young children with T1D.

Combination and advanced therapies

SAP, PLGS, and HCL therapy exist on a continuum; each combines a small pump delivering CSII with a CGM device. PLGS and $\mathrm{HCL}$ also include automated algorithms to predict low- and highsensor glucose values, with adjustment of the insulin delivery rate determined by predictions. There are no large-scale studies dedicated to understanding the efficacy of these therapies in very young children, although some larger trials have included very young children in their cohorts. Nevo-Shenker et al. ${ }^{1}$ report on one study of SAP in young children (reference no. 23); that study documented improvements in $\mathrm{HbA} 1 \mathrm{C}$ and $\mathrm{SH}$, but did not include other outcomes and did not use a valid measure of $\mathrm{FH}$. Another recent study found that transition to $\mathrm{HCL}$ improved $\mathrm{HbA} 1 \mathrm{C}$ and time in range among $<7$-year-olds. Youth in that study experienced a modest increase in time spent in the hypoglycemic range but did not experience an increase in the rate of $\mathrm{SH}^{11}$ The impact of these therapies on rates of DKA and other non-glycemic outcomes remains largely unstudied.

Research call to action

Investigators must design research to specifically target very young children and their caregivers and address the existing knowledge gaps related to their use of diabetes self-management devices. As most diabetes centers provide care for only a few dozen to a few hundred very young children, it is imperative that we build multi-center consortia to promote and expeditiously complete research that addresses the unique developmental needs of very young children. Future studies should accurately measure the unique characteristics of preschool children with T1D and their caregivers in order to determine how caregiver-child characteristics and interactions impact outcomes. Researchers and funding agencies should also evaluate whether future trials of diabetes self-management devices in these youth should be conducted for longer periods of time in order to have the power to detect treatment-related differences in low-event outcomes (e.g., SH and DKA) and to assess for longitudinal changes in adherence and efficacy. There should also be a consistent source of support for efforts to study observational data from longitudinal health outcomes data registries.

The U.S. T1D Exchange Clinic Network curated health outcomes data from over 30,000 individuals with T1D into a registry from 2010 to 2018; the registry included data from $>80$ diabetes care centers. Another U.S. registry should replace that previous effort with more cost-effective methods of data collection (e.g., automated data extraction from electronic health records [EHR]). The T1D Exchange Quality Improvement Collaborative is a nascent organization that seeks to integrate clinics' EHR data into a quality improvement registry in an effort to create a learning health system. With appropriate institutional review board review and institutional agreements, it will also be possible to use that resource to create a parallel deidentified research repository that supports observational health outcomes research. Finally, it is important that T1D registries be harmonized internationally using common data standards to allow comparative analyses of treatment outcomes across populations.

Policy call to action

The article by Nevo-Shenker et al. ${ }^{1}$ highlights the need for scrutiny and revision of state and national policies that impact families' access to new technologies to support diabetes management among young children. For example, up to 12 state Medicaid programs in the U.S. still do not cover the cost of CGM devices for children, meaning that children in those states are also excluded from the advanced therapeutics that rely on CGM as a component.
Many commercial insurers similarly exclude advanced diabetes self-management devices from coverage for children due to lack of evidence from randomized controlled trials. Policymakers and regulatory authorities should

- recognize the importance of outcomes beyond $\mathrm{HbA1c}$ in guiding decision-making related to regulatory approval, cost coverage, and access for young children with T1D. These include prevalence of $\mathrm{SH}$ and DKA, as well as $\mathrm{FH}$ and QOL among caregivers.

- consider the value of real-world evidence from large observational registries in supporting (or refuting) the efficacy and comparative effectiveness of each new therapeutic.

- acknowledge that the relative lack of funding available to support clinical trials of therapeutics in young children with T1D significantly limits the availability of evidence traditionally used by insurers to determine whether the cost of an efficacious new device is covered.

- recognize that diabetes is a unique disease in that many individuals are diagnosed in childhood, yet many of the disease's major chronic complications emerge during adulthood; this fact complicates any health economic analyses used by insurers to support coverage of the costs of new technologies in pediatric diabetes care.

- recognize that diabetes education, remote monitoring between clinic visits, remote or in-clinic behavioral interventions, and mental healthcare for parents are critical to assuring that children receive the anticipated benefit from new technologies.

\section{SUMMARY}

Researchers, regulators, and policymakers must consider the unique developmental needs of very young children with T1D and their caregivers in designing clinical trials, observational studies, and policies impacting access to new technologies.

\section{AUTHOR CONTRIBUTIONS}

M.A.C. wrote the initial commentary. S.R.P., R.J.M., and M.A. reviewed and edited for content. M.A. provided final approval.

\section{ADDITIONAL INFORMATION}

Competing interests The authors declare no competing interests.

Publisher's note Springer Nature remains neutral with regard to jurisdictional claims in published maps and institutional affiliations.

\section{REFERENCES}

1. Nevo-Shenker, M., Phillip, M., Nimri, R. \& Shalitin, S. Type 1 diabetes mellitus management in young children: implementation of current technologies. Pediatr. Res. (2019) https://doi.org/10.1038/s41390-019-0665-4. [Epub ahead of print]

2. Miller, K. M. et al. Current state of type 1 diabetes treatment in the U.S.: updated data from the T1D Exchange clinic registry. Diabetes Care 38, 971-978 (2015).

3. Perantie, D. C. et al. Effects of prior hypoglycemia and hyperglycemia on cognition in children with type 1 diabetes mellitus. Pediatr. Diabetes 9, 87-95 (2008).

4. Patton, S. R., Dolan, L. M., Henry, R. \& Powers, S. W. Parental fear of hypoglycemia: young children treated with continuous subcutaneous insulin infusion. Pediatr. Diabetes 8, 362-368 (2007).

5. Driscoll, K. A., Raymond, J., Naranjo, D. \& Patton, S. R. Fear of hypoglycemia in children and adolescents and their parents with type 1 diabetes. Curr. Diab. Rep. 16, 77 (2016)

6. Varni, J. W. et al. Diabetes management mediating effects between diabetes symptoms and health-related quality of life in adolescents and young adults with type 1 diabetes. Pediatr. Diabetes 19, 1322-1330 (2018). 
Are we there yet? Advanced technologies for young children with type $1 \ldots$ MA Clements et al.

7. Maahs, D. M. et al. Contrasting the clinical care and outcomes of 2,622 children with type 1 diabetes less than 6 years of age in the United States T1D Exchange and German/Austrian DPV registries. Diabetologia 57, 1578-1585 (2014).

8. Patton, S. R., Dolan, L. M., Henry, R. \& Powers, S. W. Fear of hypoglycemia in parents of young children with type 1 diabetes mellitus. J. Clin. Psychol. Med. Settings 15, 252-259 (2008).

9. DeSalvo, D. J. et al. Continuous glucose monitoring and glycemic control among youth with type 1 diabetes: International comparison from the T1D Exchange and DPV Initiative. Pediatr. Diabetes 19, 1271-1275 (2018).

10. Hilliard, M. E. et al. Benefits and barriers of continuous glucose monitoring in young children with type 1 diabetes. Diabetes Technol. Ther. 21, 493-498 (2019).

11. Salehi, P., Roberts, A. J. \& Kim, G. J. Efficacy and safety of real-life usage of minimed 670G automode in children with type 1 diabetes less than 7 years old. Diabetes Technol. Ther. 21, 448-451 (2019). 